\title{
Mitochondrial dysfunction and mitophagy in Parkinson's: from familial to sporadic disease
}

Brent J Ryan ${ }^{1}$, Selim Hoek ${ }^{1}$, Edward A. Fon ${ }^{2}$, and Richard Wade-Martins* ${ }^{1}$

1. Oxford Parkinson's Disease Centre, Department of Physiology, Anatomy and Genetics, Le Gros Clark Building, University of Oxford, South Parks Road, Oxford, OX1 3QX.

2.McGill Parkinson Program, Department of Neurology and Neurosurgery, Montreal Neurological Institute and Hospital, McGill University, Montréal, Québec H3A 2B4, Canada.

Correspondence to: Richard Wade-Martins

Oxford Parkinson's Disease Centre,

Department of Physiology, Anatomy and Genetics

University of Oxford, OX1 3QX, UK

Key words: Mitochondrial dysfunction, Parkinson's disease, mitophagy, oxidative stress, $\alpha$-synuclein, 


\section{Abstract}

Parkinson's disease (PD) is a progressive neurodegenerative disorder characterised by the preferential loss of dopaminergic neurons in the substantia nigra. Mitochondrial dysfunction is increasingly appreciated as a key determinant of dopaminergic neuronal susceptibility in PD and is a feature of both familial and sporadic disease, as well as in toxin-induced Parkinsonism. Recently, the mechanisms by which PD-associated mitochondrial proteins PINK1 and parkin function and induce neurodegeneration have been identified. In addition, increasing evidence implicates other PDassociated proteins such as $\alpha$-syn and LRRK2 in mitochondrial dysfunction in genetic cases of PD with the potential for a large functional overlap with sporadic disease. This review highlights how recent advances in understanding familial PD-associated proteins have identified novel mechanisms and therapeutic strategies for addressing mitochondrial dysfunction in PD. 


\section{Mitochondrial dysfunction in Parkinson's disease}

Parkinson's disease (PD) is the second most common neurodegenerative disease affecting $1 \%$ of the population over the age of 60 . The cardinal motor symptoms of PD are a result of the loss of dopaminergic neurons in the substantia nigra pars compacta ( $\mathrm{SNpc}$; see glossary) causing a deficiency of dopamine in the striatum. The exact aetiology and natural course of the disease has yet to be fully characterised but involves dysfunction of numerous systems-level processes including mitochondrial function, calcium homeostasis, dopamine homeostasis and autophagy (Box 1). Although age remains the greatest risk factor for developing sporadic PD, both common, lowpenetrance genetic variants, and rare genetic variants with large effect sizes, have helped inform us of the proteins and systems that modulate sporadic disease [1]. Familial forms of PD account for up to $10 \%$ of disease cases and usually result in early-onset disease.

Many of the PD associated genes identified by both genome-wide association studies (GWAS) and Mendelian inheritance patterns encode proteins that have been shown to either directly or indirectly play a role in mitochondrial homeostasis or mitophagy (see glossary). Amongst these mutations, the proteins phosphatase and tensin homolog deleted on chromosome 10 (PTEN)induced putative kinase 1 (PINK1), parkin (PARK2) and DJ-1 all cause autosomal recessive PD and have well defined roles in mitochondrial homeostasis and mitophagy, with PINK1 and parkin being key activators of mitophagy and DJ-1 being a redox sensor and chaperone [2]. In contrast, mutations in a number of other PD-associated proteins primarily located in the cytosol, such as leucine-rich repeat kinase 2 (LRRK2; see glossary) and $\alpha$-synuclein ( $\alpha$-syn; see glossary), cause autosomal dominant PD. Although these autosomal dominant genes have been shown to cause a wide range of cellular pathologies, they have also been shown to modulate mitochondrial function. Indeed, whilst neurons are lost from a number of regions in PD patients, it is the unique susceptibility profile of SNpc neurons which result in their loss and the associated PD symptoms (Box 2). Prominent among these susceptibility factors are bioenergetic demands, increased oxidative stress and calcium 
buffering of SNpc neurons, all of which may converge on mitochondrial function. PD-causing mutations have been demonstrated to either increase mitochondrial damage or impair clearance of damaged mitochondria resulting in cellular stress (Box 1). This review will explore recent advances in our understanding of the roles of LRRK2 and $\alpha$-syn, PINK-1, parkin and DJ-1 and how these mechanisms may be operative in sporadic PD. Finally, we will discuss how these recent findings influence the development of therapeutics targeting mitochondrial pathways.

\section{PINK1, parkin and DJ-1 as key mediators of mitochondrial homeostasis and mitophagy}

PINK1-dependent activation of parkin is recognised as a major route of mitophagy and is essential for mitochondrial quality control in a number of models. Recently, significant advances have been made in defining the precise mechanism of PINK1-dependent parkin activation (Box 3). PINK1 activity is primarily regulated by mitochondrial import across polarised mitochondrial membranes and subsequent cleavage. This import of PINK1 into mitochondria occurs via the translocase of the outer-membrane (TOM) complex and into the mitochondrial matrix via the TIM complex whereupon it is cleaved by mitochondrial processing peptidase and the presenilin-associated rhomboid-like protease (PARL) $[3,4]$. These cleaved forms of PINK1 are degraded by the cytosolic proteasome, although recently, cleaved PINK1 has been shown to act as a repressor of parkin translocation $[3,5]$. Mutations located around the A103 cleavage site in PINK1 such as C82F, Q115L and R147H, result in impaired cleavage by mitochondrial proteases and aberrant parkin recruitment [4]. Blockade of mitochondrial protein import results in PINK1-dependent parkin recruitment to TOM-complex components TOM70A and TOM40 resulting in degradation of outer mitochondrial membrane (OMM) proteins [6]. Indeed, the activation of PINK1, resulting from loss of mitochondrial membrane potential (MMP) or accumulation of misfolded proteins, is dependent on autophosphorylation and phosphorylation of ubiquitin for parkin recruitment and activation (see Box 3 for details) $[7,8]$.

Targets of activated parkin 
Once activated, parkin acts as an E3 ligase, catalysing ubiquitination of numerous substrates with diverse roles in mitochondrial homeostasis including the mitochondrial fusion regulators $M f n 1 / 2$, the fusion regulator Drp1 as well as DJ-1 [9-11]. Recently, an effort to fully map the parkindependent ubiquitylome (see glossary) has identified a number of novel parkin substrates [12]. A key parkin substrate appears to be the mitochondrial motility regulating protein Miro, which promotes anterograde mitochondrial movement via kinesin motor (KIF5) association with microtubules [13, 14]. Indeed, PINK1 knockdown results in increased anterograde mitochondrial movement, which has been suggested may be linked to peripheral neuropathy seen in PD patients with either PINK1 or parkin mutations through accumulation of damaged mitochondria in axons [14]. In support of these observations, LC3-dependent mitophagy of mitochondria damaged by ROS in distal axons of hippocampal neurons is PINK1/parkin dependent [15].

Under severe stress, elicited by valinomycin, PINK1/parkin acts as molecular switch regulating mitochondrial homeostasis and apoptosis through the ubiquitin-mediated degradation of Mcl-1 (see glossary) [16]. This observation suggests that the nature/severity of the stress is an important factor in the extent of PINK1/parkin activation and ultimately the initiation of mitophagy or apoptosis.

\section{Parkin-independent functions of PINK1}

A recent, comprehensive study of mitochondrial respiratory activities and protein abundance revealed that PINK1 KO fibroblasts show decreased complex I,III and IV activities, at least partially due to decreased complex abundances [17]. These data are consistent with previous reports showing oxidative phosphorylation (see glossary) deficits and synaptic dysfunction in both Drosophila and mice with PINK1 mutations [18]. A number of independent papers have also demonstrated mitochondrial complex I deficiencies in PINK1 knockouts and mutants. For example, mitochondrial complex I subunit NdufA10 is directly phosphorylated by PINK1 at Ser250, which is important for the ubiquinone reductase activity of complex I [19]. In fibroblasts and iPSC-derived neurons from PD patients with mutations in PINK1, phosphomimetic complex I subunit NdufA10 
rescued complex I activity and MMP [19]. Interestingly, the drosophila homologue of NdufA10 restores complex I activity and partially rescues locomotor deficits which cannot be rescued by parkin overexpression [20].

A further example of parkin-independent PINK1 activity is the observation that PINK1 but not parkin mutants can be rescued by both the GDNF receptor Ret and TNF receptor-associated protein-1 (TRAP1)[21-23]. Both Ret- and TRAP1-dependent rescue of bioenergetics and mitochondrial morphology was independent of mitophagy or parkin recruitment in Drosophila. In addition, Ret activation and TRAP1 expression rescue complex I deficiency in PINK1 but to a lesser extent in parkin mutants [21, 23]. Together, these data demonstrate the existence of a possibly convergent PINK1dependent pathway for mitochondrial homeostasis this is distinct from the PINK1-parkin axis.

\section{DJ-1- a link between mitochondrial and cytosolic dysfunction?}

DJ-1 (PARK7) has a diverse range of cellular functions, including as a redox sensor/reductase [24] and mutations in DJ-1 are known to cause familial PD. Redox regulation of DJ-1 mediates its role as a chaperone, protease, glyoxalase and regulator of transcription, influencing autophagy and mitochondrial dysfunction. Mitochondrial localisation of DJ-1 is neuroprotective and is dependent on oxidation of the free cysteine ( $\mathrm{C} 106)$ residue by ROS and the formation of a cysteine sulfinic $\left(\mathrm{SO}_{2}\right)$ acid through ROS scavenging modulates protein function [24] (Figure 1). Mitochondrial localisation of DJ-1 during oxidative stress is dependent on S to $G 2$ phase nuclear autoantigen (SG2NA) and DJ-1 associated PD-associated mutations M26I, C106A and L166P inhibit this process [25]. However, DJ-1 knockout mice show increased antioxidant enzyme activity suggesting either increased $\mathrm{H}_{2} \mathrm{O}_{2}$ production or a compensatory response to deal with lack of DJ-1 [26].

DJ-1 directly interacts with both monomeric and oligomeric $\alpha$-syn, inhibiting oligomer formation (as discussed in detail below). Additionally, this interaction protects cells from $\alpha$-syn toxicity in a yeast model [27]. Importantly, DJ-1- $\alpha$-syn interactions are disrupted by several (L10P, M26I and P158del), 
but not all, PD-linked DJ-1 mutations [27]. It is not clear yet if this interaction occurs in a specific subcellular compartment, such as in mitochondria or in response to stimuli, such as DJ-1 oxidation.

\section{Mutated and aggregated $\alpha$-synuclein as mediators of mitochondrial dysfunction}

Roles of $\alpha$-synuclein aggregation in mitochondrial dysfunction

The aggregation of $\alpha$-syn and its incorporation as a major component of Lewy bodies (see glossary) is the hallmark of Parkinson's disease. Oligomerisation/aggregation of $\alpha$-syn in response to oxidation or phosphorylation likely mediates its deleterious effects. Highly penetrant familial mutations multiplications in the $\alpha$-syn gene (SNCA) have been shown to cause aggressive early-onset PD. Additionally, a common SNP in the SNCA gene which is enriched in PD patients has been demonstrated to increase expression in vitro [28]. Furthermore, recent evidence suggests that $\alpha$-syn may play a role at the mitochondrial-associated membrane (MAM) [29] and extracellular $\alpha$-syn may act as a prion to propagate $\alpha$-syn aggregation and cell stress in PD [30], suggesting novel roles of wild-type and pathogenic $\alpha$-syn.

Multiple studies have demonstrated the effects of $\alpha$-syn overexpression/oligomerisation on mitochondria both in vitro and in vivo. These dysfunctions include inhibition of mitochondrial complexes [31], increased mitochondrial fragmentation in the presence of $\alpha$-syn oligomers in cells [32], and the permeabilization of mitochondrial-like lipid vesicles by $\alpha$-syn oligomers [33]. Interestingly, the effects of overexpression appear to be brain region- and oligomeric statedependent. For example, $\alpha$-syn overexpression induces complex I, II, IV and V inhibition in the midbrain but complex IV and V dysfunction the striatum [31]. In addition, intermediate $\alpha$-syn aggregates (pre-fibrillar forms), but not monomeric or fibrillar forms, reduce mitochondrial $\mathrm{Ca}^{2+}$ retention resulting in $\mathrm{Ca}^{2+}$ dependent mitochondrial dysfunction including loss of MMP and NADH oxidation in isolated mitochondria via complex I dysfunction [34]. The neurotoxin MPTP/MPP+ causes dopaminergic cell death through complex I inhibition and ROS production. $\alpha$-syn protein is required for MPTP induced neurodegeneration, a function retained in the mutated forms of the 
protein, potentially indicating an essential role for $\alpha$-syn in the propagation of complex I-induced dysfunction $[35,36]$. Knockdown of $\alpha$-syn also protects cells from MPP+ toxicity in vitro, potentially through mechanisms involving increased levels of the antioxidant tetrahydrobiopterin, which may serve to neutralise mitochondria-derived ROS [37].

$P D$-causing point mutation in $\alpha$-syn induce mitochondrial dysfunction

A number of PD-associated mutations in $\alpha$-syn occur in the amphipathic $\mathrm{N}$-terminal of the protein (A30P, E46K, H50Q and A53T). This region of the protein responsible for both self-interactions and interactions with lipids with varying effects of mutations observed. The recently discovered $\mathrm{H} 50 \mathrm{Q}$ mutation [38] has been demonstrated to induce only a small number of pathological effects, including oligomerisation and aggregation in SH-SY5Y cells and increased mitochondrial fragmentation in hippocampal neurons [39]. This is consistent with the observations showing increased mitochondrial fragmentation induced by fibrillar $\alpha$-syn [34]. BAC-transgenic mice expressing 2-3 fold higher E46K $\alpha$-syn demonstrate a mild neurodegenerative phenotype and increased sensitivity to rotenone-induced cell death [40]. However, overexpression of wild-type $\alpha$ syn at similar levels using BACs, has demonstrated a similar pre-clinical phenotype, but with the development of age-dependent loss of dopaminergic neurons and the onset of locomotor deficiencies, suggesting that these effects may be attributed to $\alpha$-syn overexpression [41].

The A53T mutation is the most well-studied $\alpha$-syn mutation and results in the presence of more fragmented mitochondria and increased ROS production both of which also correlate with aggregation-inducing S129 phosphorylation of $\alpha$-syn [42]. This correlation between mitochondrial phenotypes and S129 phosphorylation suggests that phosphorylated $\alpha$-syn may be an important mito-toxic $\alpha$-syn species, possibly through increasing $\alpha$-syn aggregation.

Indirect modulation of mitochondrial function by $\alpha$-syn 
Induced pluripotent stem cells (iPSC) neurons derived from A53T patients produce more ROS as well as reactive nitrogen species (RNS; see glossary) in response to stimulus, compared to isogenic control lines [43]. One of the consequences of this oxidative stress is increased oxidation and Snitrosation of the transcription factor, myocyte enhancer factor 2C (MEF2C). These posttranslational modifications result in decreased MEF2C binding to the several targets including the PGC-1 $\alpha$ promoter resulting in decreased mitochondrial biogenesis [43].

Mitochondrial associated ER membranes (MAM) are increasingly being appreciated as key regulators of many mitochondrial processes including dynamics, $\mathrm{Ca}^{2+}$ homeostasis and lipid synthesis, as previously reviewed [44]. In addition, it has been demonstrated that the MAM is a major site of syntaxin-17-dependent autophagosome formation in starved cells [45]. MAM also act as key regulatory sites of mitochondrial fission. MAM are contact sites between ER tubules and mitochondria, formed prior to Drp1 translocation representing sites of fission [46]. PD-associated mutations to $\alpha$-syn result in reduced association of $\alpha$-syn with the MAM and reduced ERmitochondrial association, concomitant with increased Drp1-independent fission, possibly though altered OPA1 processing [29]. These data suggest that increases in $\alpha$-syn levels or post-translational modifications may affect MAM.

\section{The role of increased LRRK2 kinase activity in mitochondrial dysfunction}

LRRK 2 is a multifunction protein with important kinase activities, the PD-associated mutation G2019S (kinase domain) thought to increase kinase activity of the protein [47]. Wild-type LRRK2 interacts with a number of key regulators of mitochondrial fission/fusion, co-localising with them either in the cytosol or on mitochondrial membranes, indicating it has multiple regulatory roles (Figure 2) [48, 49]. It has been established that in murine primary neurons and human neuroblastoma, endogenous LRRK2 directly interacts with Drp1 at the mitochondrial membrane, increasing Drp1 phosphorylation and mitochondrial fission [49, 50]. This LRRK2-Drp1-dependent mitochondrial fragmentation is enhanced by overexpressing wild-type LRRK2 and by expressing the 
PD-associated G2019S form of the protein but may be rescued by inhibiting Drp1 or increasing fusion $[49,51]$. Furthermore, kinase-dead or GTP-binding-deficient LRRK2 displays greatly decreased Drp1 interaction [49]. Phosphorylation of S616 of Drp1 has been shown to promote fission and increased S616 phosphorylation has been observed in sporadic PD patients [52] [53]. However, other data suggest that the G2019S mutant primarily phosphorylates Drp1 at T595 resulting in aberrant mitochondrial fragmentation [51]. LRRK2 also interacts with the mitochondrial fusion regulators Mfn1/2 and OPA1 modulating their activities; PD patients carrying the G2019S mutation demonstrate decreased levels of mature OPA1 [48]. Together, these data demonstrate that the increased kinase activity of LRRK2 results in decreased mitochondrial fusion in addition to increased fission and suggest that regulation of LRRK2 kinase activity may be an important factor in mitochondrial fission/fusion in sporadic PD.

The effects of LRRK2 mutations in patient-derived iPSCS

Expression of mutant LRRK2 and/or overexpression of wild type LRRK2 induce a variety of negative effects on mitochondrial and cellular health. These effects include increased fragmented mitochondria producing more ROS, less ATP and an increased vulnerability of cells to stressors. iPSCderived dopamine neurons generated from R1441C mutation carriers show increased oxidative stress, higher cell death and impaired neuronal differentiation [54]. Furthermore, G2019S expressing fibroblasts and $\mathrm{SH}-\mathrm{SY} 5 \mathrm{Y}$ cells show up to $80 \%$ increase in basal oxygen consumption and a marked decrease in MMP, due to mitochondrial proton leak caused by increased mitochondrial uncoupling protein 2 (UCP2) and UCP4 expression, with inhibition of LRRK2 normalising UCP4 expression [55]. Additionally, UCP2 upregulation has been observed in iPSC neurons from PD patients carrying the G2019S mutation [56].

Mature iPSC-derived LRRK2 G2019S dopamine neurons are more vulnerable to $\mathrm{H}_{2} \mathrm{O}_{2}$, 6hydroxydopamine (6-OHDA), rotenone and the proteasome inhibitor MG-132, compared to wild type LRRK2 neurons $[57,58]$. Increased susceptibility to MPP+ was also found in human LRRK2 G2019S fibroblasts, again with an increase in caspase-mediated cell death [59]. Together, these 
findings demonstrate how LRRK2 mutations increase cellular susceptibility towards environmental insults. This increased susceptibility may explain the incomplete penetrance of the LRRK2 mutations observed in PD in that low penetrance mutations such as those found in LRRK2 may require an additional environmental or genetic disposition to elicit/propagate neurodegeneration.

Protein aggregation and autophagy inhibition as catalysts for mitochondrial impairment?

Impairments of autophagy, particularly chaperone-mediated-autophagy (CMA), are a widely reported feature of both LRRK2 and $\alpha$-syn models $[60,61]$. In addition, accumulation of $\alpha$-syn, a CMA substrate, has been detected in multiple iPSC-derived models $[57,58,62]$. Given that LRRK2 has been shown to impair CMA [60], this may be a contributing factor towards increased $\alpha$-syn aggregation which may initiate $\alpha$-syn-dependent mitochondrial damage. Mitochondrial damage results in increased autophagic cargo and $\alpha$-syn aggregation, further inhibiting CMA, LRRK2 mutations may initiate a self-perpetuating insult on the cell (Figure 2). Feedback mechanisms such as this suggest potential ways in which stochastic protein aggregation, due to environmental factors or aging, and potentially common PD-associated genetic variants, may contribute to sporadic PD.

\section{Novel mitochondrial therapeutic targets}

Models of autosomal dominant and autosomal recessive forms of PD demonstrate markedly different mechanisms for the induction of mitochondrial dysfunction and cellular stress. However, the outcomes of this dysfunction- increased ROS production and bioenergetic stress- are common to all forms of mitochondrial dysfunction (Box 1). The wealth of mechanistic information on the mitochondrial functions of PD-related proteins has identified a wide range of points for therapeutic intervention. Translation of these promising therapeutic targets to both patients with familial mutations and further towards sporadic disease is a major challenge for PD research.

\section{Modulating mutated proteins directly}

The autoinhibition exhibited by parkin may also be a target for small molecules which increase parkin activation in a PINK1 independent manner [63]. Given that parkin activation requires both 
mitochondrial translocation and ubiquitin phosphorylation, as evidenced by phosphomimetic parkin [64], it will be interesting to see whether merely decreasing autoinhibition restores regulation and impacts mitochondrial phenotypes in the absence of PINK1.

Upregulation of the glyoxalase activity of DJ-1, using small molecules to increase catalytic rate/recycling of the protein, has the potential to reduce the levels of reactive aldehydes such as methylglyoxal that can damage proteins through the formation of advanced glycation endproducts (AGEs) [65]. Furthermore, the products of DJ-1 glyoxalase activity, D-lactate and glycolate, can rescue the loss of MMP observed after PINK1 knockdown suggesting an additional mechanism by which upregulation of DJ-1 glyoxalase may be protective [66].

Much interest has been generated by the modulation of the LRRK2 kinase domain as a potential therapy for LRRK2 mutation carriers. Treatment of neuronal cultures with the LRRK2 inhibitors LRRK2-IN-1 and GW5074 lead to an increased cell survival in LRRK2 G2019S iPSC dopaminergic neurons [58] and reduced cell dysfunction and death in response to valinomycin and concanamycin A treatment [67]. However, the lack of specificity of many of the inhibitors developed, particularly for G2019S LRRK2 has impaired their ability to be robust and translatable $[68,69]$.

It has been shown recently that oxidative stress promotes the uptake of $\alpha$-syn by various cell types resulting in mitochondrial inhibition $[70,71]$. This raises the possibility that mitochondrial dysfunction and oxidative stress may play a key role in the propagation of $\alpha$-syn prion-like behaviour. Interestingly, the cell to cell $\alpha$-syn transmission has recently been shown to be blocked in both primary culture and mice using antibodies targeting $\alpha$-syn, suggesting the future potential for development of vaccines targeting extracellular $\alpha$-syn [72].

\section{Modulating alternative/parallel pathways}

Expression of UCP4a in flies carrying PINK1 and parkin mutations significantly reduces but does not abolish both dysfunctional mitochondrial phenotypes and gross defects in both PINK1 and parkin 
mutants [73]. Conversely, the UCP inhibitor genepin restores MMP in G2019S LRRK2 mutants [55]. These conflicting effects of UCP modulation demonstrate that, whilst mitochondrial phenotypes elicited by PINK1 and G2019S mutations are similar, the mechanisms by which these mutations are operative are very different. Modulation of UCP responses in sporadic PD, using genepin-like compounds, would require establishment of whether activation/inhibition of UCP responses is useful in sporadic disease and furthermore, selective targeting of specific neuronal populations. Upregulation of the mitochondrial unfolded protein response (mtUPR) via NAD+ activation of sirtuin 2.1(potentially by the pentose phosphate pathway; Box 1) has been demonstrated to increase life span in C. elegans suggesting bioenergetics and mtUPR are crucial for successful aging with clear parallels to dysfunction observed in PD. These observations suggest that increasing the pentose phosphate pathway or activators of sirtuin 2.1 may have therapeutic utility in both familial and sporadic PD.

Cholesterol oximes such as olesoxime and TRO40303 are small molecules that are known to interact with OMM proteins including VDAC and limit opening of the mitochondrial transition pore in response to oxidative stress [74]. Olesoxime protects differentiated SH-SY5Y cells from $\alpha$-syn overexpression-mediated toxicity [75], whereas TRO40303 upregulates a number of mitochondriallyand PD-related genes including Drp1, VDAC and tyrosine hydroxylase in mice overexpressing $\alpha$-syn [76]. However, subtle modulation of behavioural phenotypes in TRO40303 mice suggest the effect may not be straightforward [76].

\section{Modulating the downstream consequences of mitochondrial stress}

Modulation of the downstream consequences of mitochondrial dysfunction may offer therapeutic opportunities applicable to both familial and sporadic PD. Recently, both transcriptional and metabolic changes in nucleotide metabolism were identified in Drosophila lacking Pink1 [77]. Genetic supplementation of deoxyribonucleoside kinase in Drosophila, or supplementation with deoxyribonucleosides or folic acid in human neuroblastoma cells with PINK1 knockdown, resulted in improvement in a number of markers of mitochondrial dysfunction [77]. 
ATP depletion, which is observed in models of severe mitochondrial dysfunction, may be a limiting factor for kinases, such as PINK1, in cells under bioenergetic stress. Therefore, it is interesting that the ATP analogue kinetin triphosphate (KTP) or its precursor kinetin (through KTP) are able to increase the activity of both native and mutant PINK1, resulting in increased parkin recruitment to damaged mitochondria and decreased sensitivity to oxidative stress under conditions of ATP depletion [78].

Supplementation with either the native mitochondrial complex I electron acceptor coenzyme $Q_{10}$ $\left(\mathrm{CoQ}_{10}\right)$ or a mitochondrially-targeted $\mathrm{CoQ}_{10}$ (MitoQ; $\mathrm{CoQ}_{10}$ coupled to the lipophilic molecule triphenylphosphoium) have previously been demonstrated to be beneficial in a number of preclinical models of PD. Unfortunately, phase 3 trials of high doses of both $\operatorname{CoQ}_{10}$ and MitoQ demonstrated no clinical benefit in PD patients relative to placebo $[79,80]$. Interestingly, vitamin $\mathrm{K}_{2}$ like $\mathrm{CoQ}_{10}$ acts as an electron acceptor and reduces PINK1/parkin associated phenotypes [81]. Therefore, activators of the vitamin $K_{2}$ producing enzyme UBIAD1 or supplementation may be hypothesised to exert a therapeutic effect in PD.

A library screening approach using fibroblasts from patients carrying parkin mutations identified 15 compounds that normalised MMP and ATP levels in patient cells, including ursocholanic acid which acts through glucocorticoid receptors and Akt signalling. This compound has also been shown to rescue mitochondrial phenotypes in LRRK2 patient fibroblasts [82]. This screening approach using fibroblasts and potentially iPSC neuronal cultures represents a paradigm for screening compound libraries for novel and repositionable compounds for PD. Screening of individual patient-derived iPSCs in this manner may represent a strategy for assessing the pre-clinical efficacy of individually targeted therapies. Furthermore, normalisation of robust phenotypes, such as decreased complex I activity, in iPSCs from sporadic PD patients is a logical and potentially hugely beneficial strategy for future PD therapeutics. 
PD patients with either autosomal dominant or autosomal recessive PD, or models of these mutations, demonstrate mitochondrial complex-I deficiencies, mitochondrial DNA damage and oxidative stress. However, as discussed earlier, it is likely that mitochondrial dysfunction in autosomal dominant and autosomal recessive PD does not occur by the same mechanism. Indeed, even from a clinical perspective, autosomal recessive mutations may represent a nigral-specific degeneration (nigropathy) due the selective vulnerability of the SNpc to mitochondrial stressors (as evidenced by the lack of non-motor dysfunction in PINK1/parkin patients), whereas autosomal dominant mutations in $\alpha$-syn and LRRK2 may represent synucleinopathies, which are driven by protein aggregation and autophagic impairment resulting in mitochondrial dysfunction much more broadly. Together, increased understanding of the mechanisms of mitophagy and increased understanding of how pathogenic PD mutations disrupt mitophagy have increased our understanding of both mitophagy and PD aetiology, identifying exciting novel therapeutic avenues. Our understanding of the connection between PD and mitophagy would be further advanced by answering several outstanding questions (Outstanding Questions Box). Key for the development of novel therapeutics is how observations in familial forms of PD translate into sporadic PD. The complex nature of PD and our experience with the failure of monotherapies targeting single systems in PD hint at the need for modulation of multiple causes of mitochondrial dysfunction in concert with other systems-level processes in PD. 


\section{$\underline{\text { References }}$}

1 Nalls, M.A., et al. (2014) Large-scale meta-analysis of genome-wide association data identifies six new risk loci for Parkinson's disease. Nat Genet 46, 989-993

2 Trempe, J.F. and Fon, E.A. (2013) Structure and Function of Parkin, PINK1, and DJ-1, the Three Musketeers of Neuroprotection. Frontiers in neurology 4, 38

3 Greene, A.W., et al. (2012) Mitochondrial processing peptidase regulates PINK1 processing, import and Parkin recruitment. EMBO Rep 13, 378-385

4 Deas, E., et al. (2011) PINK1 cleavage at position A103 by the mitochondrial protease PARL. Human Molecular Genetics 20, 867-879

5 Fedorowicz, M.A., et al. (2014) Cytosolic cleaved PINK1 represses Parkin translocation to mitochondria and mitophagy. EMBO Rep 15, 86-93

6 Bertolin, G., et al. (2013) The TOMM machinery is a molecular switch in PINK1 and PARK2/PARKINdependent mitochondrial clearance. Autophagy 9, 1801-1817

7 Okatsu, K., et al. (2012) PINK1 autophosphorylation upon membrane potential dissipation is essential for Parkin recruitment to damaged mitochondria. Nat Commun 3, 1016

8 Jin, S.M. and Youle, R.J. (2013) The accumulation of misfolded proteins in the mitochondrial matrix is sensed by PINK1 to induce PARK2/Parkin-mediated mitophagy of polarized mitochondria. Autophagy 9, 1750-1757

9 Chen, Y. and Dorn, G.W. (2013) PINK1-Phosphorylated Mitofusin 2 Is a Parkin Receptor for Culling Damaged Mitochondria. Science 340, 471-475

10 Wang, H., et al. (2011) Parkin Ubiquitinates Drp1 for Proteasome-dependent Degradation: Implication of Dysregulated Mitochondrial Dynamics in Parkinson's Disease. Journal of Biological Chemistry 286, 11649-11658

11 Geisler, S., et al. (2010) PINK1/Parkin-mediated mitophagy is dependent on VDAC1 and p62/SQSTM1. Nat Cell Biol 12, 119-131

12 Sarraf, S.A., et al. (2013) Landscape of the PARKIN-dependent ubiquitylome in response to mitochondrial depolarization. Nature 496, 372-376

13 Wang, X., et al. (2011) PINK1 and Parkin target Miro for phosphorylation and degradation to arrest mitochondrial motility. Cell 147, 893-906

14 Liu, S., et al. (2012) Parkinson's Disease-Associated Kinase PINK1 Regulates Miro Protein Level and Axonal Transport of Mitochondria. PLoS Genet 8, e1002537

15 Ashrafi, G., et al. (2014) Mitophagy of damaged mitochondria occurs locally in distal neuronal axons and requires PINK1 and Parkin. The Journal of Cell Biology 206, 655-670

16 Zhang, C., et al. (2014) PINK1 Triggers Autocatalytic Activation of Parkin to Specify Cell Fate Decisions. Current Biology 24, 1854-1865

17 Amo, T., et al. (2014) Detailed analysis of mitochondrial respiratory chain defects caused by loss of PINK1. Neuroscience Letters 580, 37-40

18 Morais, V.A., et al. (2009) Parkinson's disease mutations in PINK1 result in decreased Complex I activity and deficient synaptic function. EMBO molecular medicine 1, 99-111

19 Morais, V.A., et al. (2014) PINK1 Loss of Function Mutations Affect Mitochondrial Complex I Activity via NdufA10 Ubiquinone Uncoupling. Science 344, 203-207

20 Pogson, J.H., et al. (2014) The Complex I Subunit <italic $>$ NDUFA10</italic> Selectively Rescues $<$ italic>Drosophila pink1</italic> Mutants through a Mechanism Independent of Mitophagy. PLoS Genet 10, e1004815

21 Costa, A.C., et al. (2013) Drosophila Trap1 protects against mitochondrial dysfunction in a PINK1/parkin model of Parkinson/'s disease. Cell Death Dis 4, e467

22 Zhang, L., et al. (2013) TRAP1 rescues PINK1 loss-of-function phenotypes. Human Molecular Genetics 22, 2829-2841

23 Klein, P., et al. (2014) Ret rescues mitochondrial morphology and muscle degeneration of Drosophila Pink1 mutants. EMBO J 33, 341-355 
24 Canet-Aviles, R.M., et al. (2004) The Parkinson's disease protein DJ-1 is neuroprotective due to cysteine-sulfinic acid-driven mitochondrial localization. Proc Natl Acad Sci U S A 101, 9103-9108

25 Tanti, G.K. and Goswami, S.K. (2014) SG2NA recruits DJ-1 and Akt into the mitochondria and membrane to protect cells from oxidative damage. Free Radical Biology and Medicine 75, 1-13

26 Lopert, P. and Patel, M. (2014) Brain mitochondria from DJ-1 knockout mice show increased respiration-dependent hydrogen peroxide consumption. Redox biology 2, 667-672

27 Zondler, L., et al. (2014) DJ-1 interactions with [alpha]-synuclein attenuate aggregation and cellular toxicity in models of Parkinson/'s disease. Cell Death Dis 5, e1350

28 Mizuta, l., et al. (2013) YY1 binds to [alpha]-synuclein 3[prime]-flanking region SNP and stimulates antisense noncoding RNA expression. J Hum Genet 58, 711-719

29 Guardia-Laguarta, C., et al. (2014) alpha-Synuclein Is Localized to Mitochondria-Associated ER Membranes. Journal of Neuroscience 34, 249-259

30 Hansen, C., et al. (2011) $\alpha$-Synuclein propagates from mouse brain to grafted dopaminergic neurons and seeds aggregation in cultured human cells. The Journal of Clinical Investigation 121, 715-725

31 Subramaniam, S.R., et al. (2014) Region specific mitochondrial impairment in mice with widespread overexpression of alpha-synuclein. Neurobiology of Disease 70, 204-213

32 Plotegher, N., et al. (2014) Number and Brightness analysis of alpha-synuclein oligomerization and the associated mitochondrial morphology alterations in live cells. Biochimica et Biophysica Acta (BBA) - General Subjects 1840, 2014-2024

33 Stefanovic, A.N.D., et al. (2014) $\alpha$-Synuclein oligomers distinctively permeabilize complex model membranes. FEBS Journal 281, 2838-2850

34 Luth, E.S., et al. (2014) Soluble, Prefibrillar $\alpha$-Synuclein Oligomers Promote Complex I-dependent, Ca2+-induced Mitochondrial Dysfunction. Journal of Biological Chemistry 289, 21490-21507

35 Taylor, T.N., et al. (2014) Region-specific deficits in dopamine, but not norepinephrine, signaling in a novel A3OP alpha-synuclein BAC transgenic mouse. Neurobiol Dis 62, 193-207

36 Fountaine, T.M., et al. (2008) The effect of alpha-synuclein knockdown on MPP+ toxicity in models of human neurons. Eur J Neurosci 28, 2459-2473

37 Ryan, B.J., et al. (2014) alpha-Synuclein and mitochondrial bioenergetics regulate tetrahydrobiopterin levels in a human dopaminergic model of Parkinson disease. Free Radic Biol Med 67, 58-68

38 Appel-Cresswell, S., et al. (2013) Alpha-synuclein p.H50Q, a novel pathogenic mutation for Parkinson's disease. Movement Disorders 28, 811-813

39 Khalaf, O., et al. (2014) The H50Q Mutation Enhances $\alpha$-Synuclein Aggregation, Secretion, and Toxicity. Journal of Biological Chemistry 289, 21856-21876

40 Cannon, J.R., et al. (2013) Expression of human E46K-mutated $\alpha$-synuclein in BAC-transgenic rats replicates early-stage Parkinson's disease features and enhances vulnerability to mitochondrial impairment. Experimental neurology 240, 44-56

41 Janezic, S., et al. (2013) Deficits in dopaminergic transmission precede neuron loss and dysfunction in a new Parkinson model. Proc Natl Acad Sci U S A 110, 4016-4025

42 Perfeito, R., et al. (2014) Linking alpha-synuclein phosphorylation to reactive oxygen species formation and mitochondrial dysfunction in SH-SY5Y cells. Molecular and Cellular Neuroscience 62, 51-59

43 Ryan, S.D., et al. (2013) Isogenic human iPSC Parkinson's model shows nitrosative stress-induced dysfunction in MEF2-PGC1alpha transcription. Cell 155, 1351-1364

44 Vance, J.E. (2014) MAM (mitochondria-associated membranes) in mammalian cells: Lipids and beyond. Biochimica et Biophysica Acta (BBA) - Molecular and Cell Biology of Lipids 1841, 595-609

45 Hamasaki, M., et al. (2013) Autophagosomes form at ER-mitochondria contact sites. Nature 495, 389-393

46 Friedman, J.R., et al. (2011) ER tubules mark sites of mitochondrial division. Science 334, 358-362 
47 Smith, W.W., et al. (2006) Kinase activity of mutant LRRK2 mediates neuronal toxicity. Nature neuroscience 9, 1231-1233

48 Stafa, K., et al. (2014) Functional interaction of Parkinson's disease-associated LRRK2 with members of the dynamin GTPase superfamily. Hum Mol Genet 23, 2055-2077

49 Wang, X., et al. (2012) LRRK2 regulates mitochondrial dynamics and function through direct interaction with DLP1. Human Molecular Genetics 21, 1931-1944

$50 \mathrm{Niu}, \mathrm{J}$. , et al. (2012) Leucine-rich repeat kinase 2 disturbs mitochondrial dynamics via Dynaminlike protein. J Neurochem 122, 650-658

$51 \mathrm{Su}$, Y.-C. and Qi, X. (2013) Inhibition of excessive mitochondrial fission reduced aberrant autophagy and neuronal damage caused by LRRK2 G2019S mutation. Human Molecular Genetics 22, 4545-4561

52 Chang, C.-R. and Blackstone, C. (2010) Dynamic regulation of mitochondrial fission through modification of the dynamin-related protein Drp1. Annals of the New York Academy of Sciences $1201,34-39$

53 Santos, D., et al. (2014) The Impact of Mitochondrial Fusion and Fission Modulation in Sporadic Parkinson's Disease. Mol Neurobiol, 1-14

54 Bahnassawy, L., et al. (2013) The parkinson's disease-associated LRRK2 mutation R1441G inhibits neuronal differentiation of neural stem cells. Stem cells and development 22, 2487-2496

55 Papkovskaia, T.D., et al. (2012) G2019S leucine-rich repeat kinase 2 causes uncoupling proteinmediated mitochondrial depolarization. Hum Mol Genet 21, 4201-4213

56 Grunewald, A., et al. (2014) Does uncoupling protein 2 expression qualify as marker of disease status in LRRK2-associated Parkinson's disease? Antioxid Redox Signal 20, 1955-1960

57 Nguyen, Ha N., et al. (2011) LRRK2 Mutant iPSC-Derived DA Neurons Demonstrate Increased Susceptibility to Oxidative Stress. Cell Stem Cell 8, 267-280

58 Reinhardt, P., et al. (2013) Genetic correction of a LRRK2 mutation in human iPSCs links parkinsonian neurodegeneration to ERK-dependent changes in gene expression. Cell Stem Cell 12, 354-367

59 Yakhine-Diop, S.M., et al. (2014) G2019S LRRK2 mutant fibroblasts from Parkinson's disease patients show increased sensitivity to neurotoxin 1-methyl-4-phenylpyridinium dependent of autophagy. Toxicology 324, 1-9

60 Orenstein, S.J., et al. (2013) Interplay of LRRK2 with chaperone-mediated autophagy. Nature neuroscience 16, 394-406

61 Bravo-San Pedro, J., et al. (2013) The LRRK2 G2019S mutant exacerbates basal autophagy through activation of the MEK/ERK pathway. Cell Mol Life Sci 70, 121-136

62 Sanchez-Danes, A., et al. (2012) Disease-specific phenotypes in dopamine neurons from human iPS-based models of genetic and sporadic Parkinson's disease. EMBO molecular medicine 4, 380-395

63 Trempe, J.F., et al. (2013) Structure of parkin reveals mechanisms for ubiquitin ligase activation. Science 340, 1451-1455

64 Koyano, F., et al. (2014) Ubiquitin is phosphorylated by PINK1 to activate parkin. Nature 510, $162-$ 166

65 Lee, J.Y., et al. (2012) Human DJ-1 and its homologs are novel glyoxalases. Hum Mol Genet 21, 3215-3225

66 Toyoda, Y., et al. (2014) Products of the Parkinson's disease-related glyoxalase DJ-1, D-lactate and glycolate, support mitochondrial membrane potential and neuronal survival. Biology Open 3, 777784

67 Cooper, O., et al. (2012) Pharmacological rescue of mitochondrial deficits in iPSC-derived neural cells from patients with familial Parkinson's disease. Science translational medicine 4, 141ra190

68 Kramer, T., et al. (2012) Small molecule kinase inhibitors for LRRK2 and their application to Parkinson's disease models. ACS Chem Neurosci 3, 151-160

69 Liu, M., et al. (2013) Type II kinase inhibitors show an unexpected inhibition mode against Parkinson's disease-linked LRRK2 mutant G2019S. Biochemistry 52, 1725-1736 
70 Luk, K.C., et al. (2012) Pathological $\alpha$-Synuclein Transmission Initiates Parkinson-like Neurodegeneration in Nontransgenic Mice. Science 338, 949-953

71 Pukass, K. and Richter-Landsberg, C. (2014) Oxidative stress promotes uptake, accumulation, and oligomerization of extracellular alpha-synuclein in oligodendrocytes. Journal of molecular neuroscience : $M N$ 52, 339-352

72 Tran, Hien T., et al. (2014) $\alpha$-Synuclein Immunotherapy Blocks Uptake and Templated Propagation of Misfolded $\alpha$-Synuclein and Neurodegeneration. Cell Reports 7, 2054-2065

$73 \mathrm{Wu}, \mathrm{K}$., et al. (2014) UCP4A protects against mitochondrial dysfunction and degeneration in pink1/parkin models of Parkinson's disease. The FASEB Journal 28, 5111-5121

74 Bordet, T., et al. (2007) Identification and Characterization of Cholest-4-en-3-one, Oxime (TRO19622), a Novel Drug Candidate for Amyotrophic Lateral Sclerosis. Journal of Pharmacology and Experimental Therapeutics 322, 709-720

75 Gouarné, C., et al. (2015) Protective role of olesoxime against wild type alpha-synuclein-induced toxicity in human neuronally differentiated SHSY-5Y cells. British Journal of Pharmacology 172, 235245

76 Richter, F., et al. (2014) Chronic administration of cholesterol oximes in mice increases transcription of cytoprotective genes and improves transcriptome alterations induced by alphasynuclein overexpression in nigrostriatal dopaminergic neurons. Neurobiology of Disease 69, 263275

77 Tufi, R., et al. (2014) Enhancing nucleotide metabolism protects against mitochondrial dysfunction and neurodegeneration in a PINK1 model of Parkinson's disease. Nat Cell Biol 16, 157166

78 Hertz, N.T., et al. (2013) A neo-substrate that amplifies catalytic activity of parkinson's-diseaserelated kinase PINK1. Cell 154, 737-747

79 The Parkinson Study Group, Q.E.I. (2014) A randomized clinical trial of high-dosage coenzyme q10 in early parkinson disease: No evidence of benefit. JAMA Neurology 71, 543-552

80 Snow, B.J., et al. (2010) A double-blind, placebo-controlled study to assess the mitochondriatargeted antioxidant MitoQ as a disease-modifying therapy in Parkinson's disease. Movement Disorders 25, 1670-1674

81 Vos, M., et al. (2012) Vitamin K2 Is a Mitochondrial Electron Carrier That Rescues Pink1 Deficiency. Science 336, 1306-1310

82 Mortiboys, H., et al. (2013) Ursocholanic acid rescues mitochondrial function in common forms of familial Parkinson's disease. Brain : a journal of neurology 136, 3038-3050

83 Dunn, L., et al. (2014) Dysregulation of glucose metabolism is an early event in sporadic Parkinson's disease. Neurobiol Aging 35, 1111-1115

84 Brand, M.D., et al. (2004) Mitochondrial superoxide: production, biological effects, and activation of uncoupling proteins. Free Radical Biology and Medicine 37, 755-767

85 Schmid, A.W., et al. (2013) Alpha-synuclein Post-translational Modifications as Potential Biomarkers for Parkinson Disease and Other Synucleinopathies. Molecular \& Cellular Proteomics 12, 3543-3558

86 Ryan, B.J., et al. (2014) Oxidative post-translational modifications and their involvement in the pathogenesis of autoimmune diseases. Redox biology 2, 715-724

$87 \mathrm{Kim}, \mathrm{C}$. , et al. (2013) Neuron-released oligomeric $\alpha$-synuclein is an endogenous agonist of TLR2 for paracrine activation of microglia. Nat Commun 4, 1562

88 Bjorkblom, B., et al. (2013) Parkinson disease protein DJ-1 binds metals and protects against metal-induced cytotoxicity. J Biol Chem 288, 22809-22820

89 Hunn, B.H.M., et al. (2015) Impaired intracellular trafficking defines early Parkinson's disease. Trends in Neurosciences

90 Chan, C.S., et al. (2007) 'Rejuvenation' protects neurons in mouse models of Parkinson's disease. Nature 447, 1081-1086 
91 Guzman, J.N., et al. (2010) Oxidant stress evoked by pacemaking in dopaminergic neurons is attenuated by DJ-1. Nature 468, 696-700

92 Gandhi, S., et al. (2009) PINK1-Associated Parkinson's Disease Is Caused by Neuronal Vulnerability to Calcium-Induced Cell Death. Molecular Cell 33, 627-638

93 Surmeier, D.J. and Schumacker, P.T. (2013) Calcium, Bioenergetics, and Neuronal Vulnerability in Parkinson's Disease. Journal of Biological Chemistry 288, 10736-10741

94 Bolam, J.P. and Pissadaki, E.K. (2012) Living on the edge with too many mouths to feed: why dopamine neurons die. Movement disorders : official journal of the Movement Disorder Society 27, 1478-1483

95 Pissadaki, E.K. and Bolam, J.P. (2013) The Energy Cost of Action Potential Propagation in Dopamine Neurons: Clues to Susceptibility in Parkinson's Disease. Frontiers in Computational Neuroscience 7

96 Schapira, A.H.V., et al. (1989) Mitochondrial complex I deficiency in Parkinson's disease. The Lancet 333, 1269

97 Reeve, A., et al. (2013) The impact of pathogenic mitochondrial DNA mutations on substantia nigra neurons. J Neurosci 33, 10790-10801

98 Sanders, L.H., et al. (2014) Mitochondrial DNA damage: Molecular marker of vulnerable nigral neurons in Parkinson's disease. Neurobiology of Disease 70, 214-223

99 Girotto, S., et al. (2012) Dopamine-derived Quinones Affect the Structure of the Redox Sensor DJ1 through Modifications at Cys-106 and Cys-53. Journal of Biological Chemistry 287, 18738-18749

100 Grenier, K., et al. (2014) Short Mitochondrial ARF Triggers Parkin/PINK1-Dependent Mitophagy. Journal of Biological Chemistry 289, 29519-29530

101 Matsuda, N., et al. (2010) PINK1 stabilized by mitochondrial depolarization recruits Parkin to damaged mitochondria and activates latent Parkin for mitophagy. J Cell Biol 189, 211-221

102 Kazlauskaite, A., et al. (2014) Parkin is activated by PINK1-dependent phosphorylation of ubiquitin at Ser65. Biochem J 460, 127-139

103 Kane, L.A., et al. (2014) PINK1 phosphorylates ubiquitin to activate Parkin E3 ubiquitin ligase activity. The Journal of Cell Biology 205, 143-153

104 Ordureau, A., et al. (2014) Quantitative Proteomics Reveal a Feedforward Mechanism for Mitochondrial PARKIN Translocation and Ubiquitin Chain Synthesis. Molecular Cell 56, 360-375

105 Soubannier, V., et al. (2012) A vesicular transport pathway shuttles cargo from mitochondria to lysosomes. Current biology : CB 22, 135-141

106 McLelland, G.L., et al. (2014) Parkin and PINK1 function in a vesicular trafficking pathway regulating mitochondrial quality control. EMBO J 33, 282-295

107 Soubannier, V., et al. (2012) Reconstitution of mitochondria derived vesicle formation demonstrates selective enrichment of oxidized cargo. PLoS One 7, e52830

108 Bender, A., et al. (2013) TOM40 Mediates Mitochondrial Dysfunction Induced by $\alpha$-Synuclein Accumulation in Parkinson's Disease. PLoS ONE 8, e62277

$109 \mathrm{Chu}, \mathrm{Y}$., et al. (2014) Abnormal alpha-synuclein reduces nigral voltage-dependent anion channel 1 in sporadic and experimental Parkinson's disease. Neurobiol Dis 69, 1-14

$110 \mathrm{Chu}$, C.T., et al. (2013) Cardiolipin externalization to the outer mitochondrial membrane acts as an elimination signal for mitophagy in neuronal cells. Nat Cell Biol 15, 1197-1205

111 Zigoneanu, I.G., et al. (2012) Interaction of $\alpha$-synuclein with vesicles that mimic mitochondrial membranes. Biochimica et Biophysica Acta (BBA) - Biomembranes 1818, 512-519

112 Robotta, M., et al. (2014) Alpha-Synuclein Binds to the Inner Membrane of Mitochondria in an a-Helical Conformation. ChemBioChem 15, 2499-2502

113 Cole, N.B., et al. (2008) Mitochondrial translocation of $\alpha$-synuclein is promoted by intracellular acidification. Experimental Cell Research 314, 2076-2089 


\section{$\underline{\text { Acknowledgements }}$}

The authors acknowledge funding support from the Monument Discovery Award from Parkinson's UK and Parkinson's UK grants G-0801 and G-1003. 


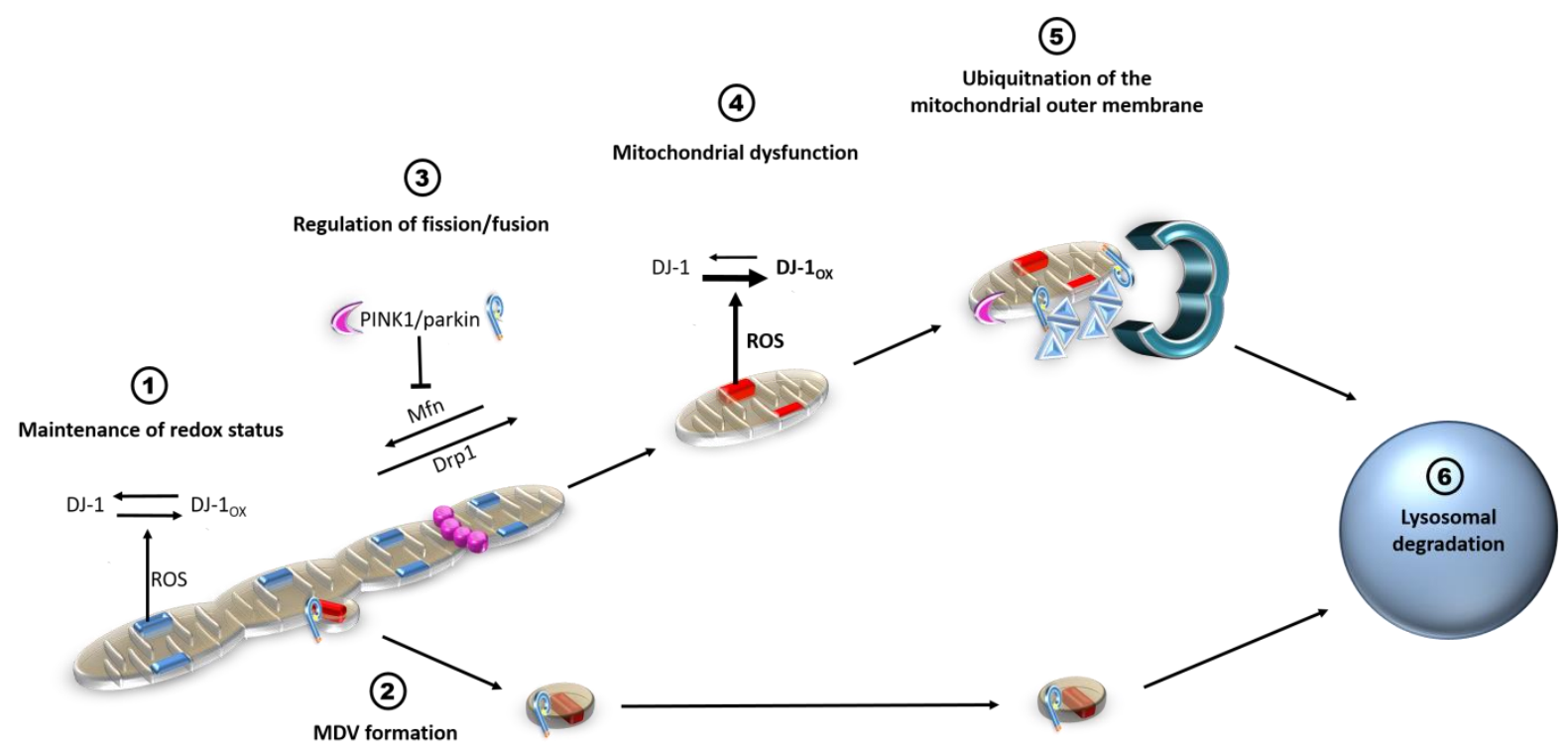

Figure 1. Overview of the roles of PD-associated mitochondrial proteins in mitochondrial homeostasis and mitophagy. 1) DJ-1 acts as a redox sensor and antioxidant in mitochondria during normal homeostasis. 2) Upon oxidative stress selective sequestration of mitochondrial components into PINK1/parkin-dependent mitochondria-derived vesicles (MDVs) occurs. These MDVs, which can fuse with lysosomes, likely act as an important mitochondrial quality-control mechanism. 3) Mitochondrial dynamics are regulated by the proteins including mitofusins (Mfn) which promote fusion and and dynamin related protein-1 (Drp1) which promotes fission. Mitochondrial fission as a result of phosphorylation (activation) of Drp1 leads to increased fragmented mitochondria which generate more ROS and less ATP. Increased ROS production causes post-translational modification of proteins including oxidation of DJ-1 promoting mitochondrial fission and degradation. DJ-1 mutations lead to excessive fission/degradation. 4) Increased ROS production by damaged mitochondria (particularly complex I) results in increased DJ-1 oxidation. 5) Loss of mitochondrial membrane potential results in PINK1-dependent recruitment and activation of parkin. Parkin acts as an E3 ligase ubiqutinylating mitochondrial proteins, particularly outer-membrane proteins, resulting in sequestration in autophagic vacuoles and 6) degradation of cargo by lysosomal proteases. 
(1)

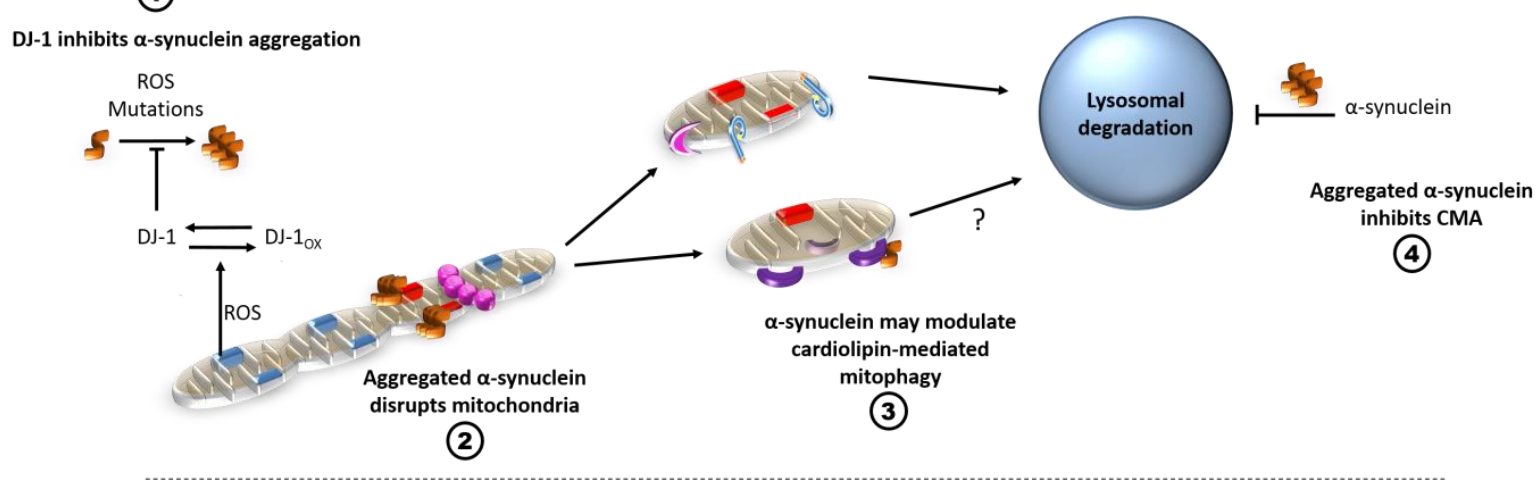

(5)

LRRK2 mutations cause aberrant DRP1 activation

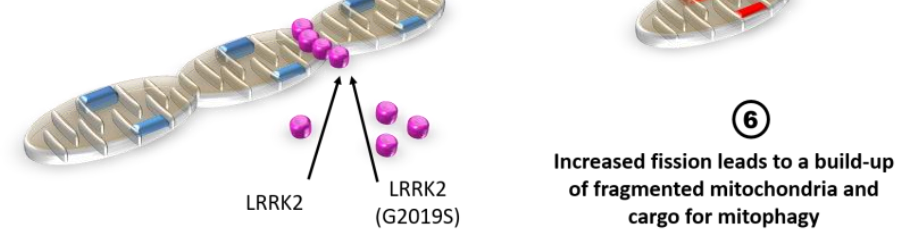

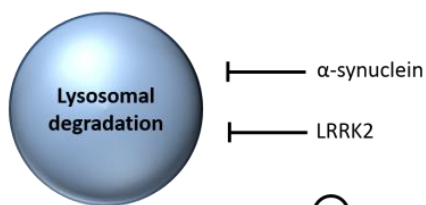

(7)

Both LRRK2 and aggregated can inhibit CMA- resulting in increased stress

Figure 2. Overview of the roles of $\alpha$-synuclein and LRRK2 in mitochondrial dysfunction. 1) Reduced but not oxidised DJ-1 inhibits $\alpha$-syn aggregation through chaperone-like activity. 2) Aggregated $\alpha$-syn may directly permeablize lipid membranes and inhibit mitochondrial complex activities. 3) Damaged mitochondria may be degraded by the PINK1/parkin pathway, however, cardiolipin externalisation on the mitochondrial surface may represent an alternative pathway. $\alpha$-synuclein binds cardiolipin suggesting it may modulate cardiolipin-mediated mitophagy. 4) Aggregated $\alpha$-synuclein inhibits Iysosomal function exacerbating cellular stress. 5) LRRK2 plays a homeostatic role in activating the mitochondrial fission protein Drp1 through its phosphorylation, however increased kinase activity results in aberrant Drp1 phosphorylation and fission. 6) Increased fission in G2019S models is associated with mitochondrial dysfunction and increased ROS production from mitochondrial complexes. The increased number of fragmented mitochondria will increase autophagic flux due to the increase in mitophagic cargo. 7) Aggregated $\alpha$-synuclein and LRRK2 mutations have been shown to impair CMA resulting in increased protein aggregation and further cellular stress. 


\section{Biochemical consequences of mitochondrial dysfunction (Box 1)}

Impairments to mitochondrial dynamics or direct inhibition of mitochondrial proteins have a number of deleterious consequences including decreased ATP generation, increased oxidant generation and cytochrome c leakage. Numerous examples of bioenergetic abnormalities in PD have been documented including complex I dysfunction and a dysregulation in glucose metabolism, impairing the pentose phosphate pathway [83]. Dysfunctional mitochondria are a reservoir for oxidant production, with complex I previously shown to be the major site of their generation [84]. Oxidative stress defines a state of oxidant production above the antioxidant capacity of the cell/tissue and results in damage of proteins, lipids and DNA with deleterious consequences. Mitochondrial reactive oxygen species (ROS) generation will result in increased mtDNA mutations or catalytic peroxidation of lipid membranes, including mitochondrial membranes which results in decreased MMP.

Oxidative post-translational modification of proteins by ROS/RNS has been demonstrated to affect the structure, function and antigenicity of proteins, such as $\alpha$-syn, in a number of diseases involving inflammation, including PD $[85,86]$. It is likely that when PD-associated proteins are posttranslationally modified by ROS that it can recapitulate the effects of loss-of-function mutations or toxic gain-of-function mutations (e.g. aggregation of $\alpha$-syn). The pathological importance of oxidant production is typified by the effects of the complex I inhibitors MPP+, rotenone and paraquat, all of which have a well described ability to induce cellular dysfunction and Parkinsonism in humans and animal models.

Increased generation of ROS/RNS in PD may be due to modulation of MMP and oxidative phosphorylation (as proposed for PINK1 and $\alpha$-syn), or produced during the respiratory burst by enzymes such as NADPH oxidase and iNOS. Indeed, inflammatory processes, such as the respiratory burst, in response to immune activation by cell debris released from dying cells or potentially via TLR recognition of aggregated $\alpha$-syn are a potentially large source of reactive species [87]. The cellular effects of this oxidant production are several, including activation of the antioxidant response via 
NRF2 and upregulation of uncoupling proteins (UCPs) that act to 'slow-down' oxidative phosphorylation and so limit oxidative phosphorylation derived ROS. The levels of ROS/RNS can be increased due to decreased antioxidant capacity (as with a number of DJ-1 mutations [88]). Therefore, by a number of different mechanisms, ROS/RNS may be produced and damage cells or inhibit cellular processes, generating more oxidative stress and cellular damage, eventually resulting in end-stage processes including apoptosis and necrosis of neurons. 


\section{Roles of mitochondria in the preferential vulnerability of dopaminergic neurons (Box 2)}

The basis for the preferential vulnerability of $A 9$ dopaminergic neurons in PD remains unresolved. However, a number of factors including increased calcium fluxes, long unmyelinated axons with large energetic demands and increased oxidative stress, contribute to the vulnerability of these neurons [89]. Whilst none of these factors is unique to A9 dopaminergic neurons, it is likely that the interplay between these factors is responsible for neurodegeneration in both familial and sporadic PD.

Neurophysiologically, it has been demonstrated that A9 dopaminergic neurons use calcium for their pacemaking activity via $\mathrm{Ca}_{\mathrm{v}} 1.3$ channels and that such pacemaking using these L-type calcium channels increases mitochondrial oxidative stress $[90,91]$. This link between $\mathrm{Ca}^{2+}$ fluxes and mitochondria is enhanced by previous demonstration that loss of the mitochondrial regulatory protein PINK1 sensitises cells to $\mathrm{Ca}^{2+}$-induced cytotoxicity [92] (as reviewed in detail [93]). The result of these fluxes, if not buffered, is disruption of MMP, increased oxidative and endoplasmic reticulum stress.

Analysis of A9 dopaminergic neuroanatomy suggests that the bioenergetic cost of both maintaining the large number of synapses and propagating action potentials is vast in long, unmyelinated dopaminergic neurons is extreme $[94,95]$. Conservative theoretical calculations, based on existing experimental data, suggest that a single midbrain dopaminergic neuron may form over one million synapses and that the energy cost of maintaining these synapses and the cellular membrane potential increases with a non-linear relationship [94, 95]. Indeed, mitochondrial complex I deficiency is a key feature of both sporadic and familial disease [96] and the non-neuronal specific complex-I inhibitor rotenone causes parkinsonism-like phenotypes in both humans and animal models. As mitochondria are a chief source of ROS more flux through mitochondria, this bioenergetic demand places strains on both the antioxidant and 
A major source of mitochondrial deficits during aging is mutations in mitochondrial DNA (mtDNA), which are accelerated by oxidative stress. Individuals carrying polymerase gamma (POLG) mutations, the polymerase responsible for mtDNA maintenance and replication, or rats treated with rotenone, acquire mtDNA deletions at an accelerated rate in the SNpc. mtDNA mutations are concomitant with decreased complex I activity and $\alpha$-syn pathology $[97,98]$, further demonstrating the susceptibility of the SNpc to mitochondrial dysfunction.

The presence of dopamine in A9 neurons is also a key susceptibility factor. Dopamine may generate ROS and also form adducts with proteins involved in mitochondrial homeostasis, such as DJ-1, impairing their function [99]. Thus dopamine provides an additional source of stress-inducing ROS if dopamine-uptake and release are not regulated effectively, as observed in both familial and sporadic PD. 


\section{Endogenous activation mechanisms of PINK1/parkin (Box 3)}

An outstanding question in PINK1/parkin biology has been the physiological relevance of the mitochondrial uncouplers, such as CCCP, which collapse MMP to induce PINK1/parkin mediated mitophagy. Recently, the endogenous molecules short mitochondrial isoform of p19ARF has been demonstrated to elicit PINK1/parkin-dependent mitophagy in neurons, highlighting that this process can be activated endogenously without exogenous manipulation of MMP [100]. In vivo, endogenous activators of PINK1/parkin are likely to include unfolded protein responses and physiological factors which reduce MMP such as $\mathrm{Ca}^{2+}$ fluxes and acidification of the cytosol.

Prior to 2014, it was known that upon loss of MMP, PINK1-dependent phosphorylation of parkin is essential for its activation. However, introducing phosphomimetic substitutions in parkin is insufficient to elicit either its activation or mitophagy in the absence of PINK1 [101]. Recently, it has been determined that PINK1 acts as an ubiquitin kinase to phosphorylate ubiquitin, which subsequently reverses autoinhibition of parkin's catalytic C431 residue $[16,64]$. These papers demonstrate that both ubiquitin and the ubiquitin like domain (UBL) of parkin is only phosphorylated at S65 in the presence of PINK1 and that this phosphorylation occurs after loss of MMP $[16,64,102,103]$. Furthermore it has been demonstrated that phosphomimetic ubiquitin activates phosphomimetic parkin, in the absence of PINK1 and that phospho-ubiquitin and parkin physically interact [64]. Furthermore quantitative proteomics data has suggested that PINK1 phosphorylates ubiquitin chains bound to mitochondrial substrates promoting further parkin activation and ubiquitylation [104]. 


\section{Mitochondrial outer-membrane- a key determinant of mitochondrial fate? (Box 4)}

In response to oxidative stress, mitochondria-derived vesicles (MDVs) are formed and selectively sequester mitochondrial proteins from the matrix and inner mitochondrial membrane $[105,106]$. These vesicles are enriched for oxidised proteins however, the source of the ROS is a key determinant in the nature of the cargo with intra-mitochondrial ROS (generated by antimycin A treatment) forming vesicles positive for mitochondrial matrix components but negative for the TOM20. In contrast, extra-mitochondrial ROS (generated by xanthine/xanthine oxidase system), generates matrix/TOM20 ${ }^{+}$MDVs in a parkin-independent manner [105]. Therefore, MDVs appear to act as a mitochondrial quality control mechanism, possibly as an alternative to degradation of the whole mitochondrion by mitophagy.

Parkin mutations disrupting the ligase active site (C431F) or translocation (K211N) of parkin abolish MDV formation, whereas mutation in the UBL domain (R42P) reduces vesicle number, potentially though impaired trafficking [106] (Figure 1). These recent studies suggest that both the TOM complex and another OMM protein- voltage-dependent anion-selective channel (VDAC)- act as key mediators of PINK1/parkin-dependent mitophagy and MDV formation. These observations are of interest, given TOM40/TOM22 levels inversely correlate with OMM degradation $[6,107]$, that $\alpha$-syn decreases TOM40 levels in mice and that VDAC1 is decreased in neurons displaying $\alpha$-syn aggregation and in PD patients $[108,109]$. Furthermore, rats expressing the PD-associated $\alpha$-syn A30P mutant demonstrated less VDAC throughout the nigra, independent of $\alpha$-syn oligomeric state [109]. However, at present, literature would favour OMM protein loss as being a consequence of $\alpha-$ syn -induced dysfunction rather than direct blockage of protein import by $\alpha$-syn.

The phospholipid cardiolipin is an integral component of the mitochondrial inner membrane which is externalised on mitochondria after stress where it acts as an "eat me" signal that is recognised by LC3, facilitating mitophagy [110]. Interestingly, $\alpha$-syn has been demonstrated to interact with cardiolipin on vesicles mimicking mitochondrial inner membranes and the A30P substitution inhibits 
this interaction $[111,112]$. Furthermore $\alpha$-syn has been shown to translocate to mitochondria after acidification of the cytosol (reducing MMP) [113]. Taken together, these data suggest that $\alpha$-syn may be recruited to cardiolipin decorating the $\mathrm{OMM}$, thus facilitating removal of damaged mitochondria. These recent observations suggest a number of mechanisms by which the mitochondrial outermembrane acts an important modulator of mitochondrial homeostasis and degradation. 


\section{Outstanding questions}

1. What are the endogenous triggers for the three main pathways of mitophagy: MDV formation, PINK1/Parkin and cardiolipin externalisation? Do these triggers determine the mechanism of degradation and how do these pathways interconnect?

2. What is the role of the MAM in regulation of mitochondrial function and how do PDassociated proteins influence this association?

3. Do mitochondrial dysfunction and oxidative stress play a significant role in promoting prionlike behaviour of $\alpha$-syn and do these two phenomena form part of a positive feedback loop in $\mathrm{PD}$ ?

4. Can modulation of mitochondrial dysfunction in isolation be sufficient to provide therapeutic benefit in symptomatic PD patients?

5. How do mitochondrial phenotypes observed in familial PD translate to sporadic PD? Is sporadic PD driven by SNPs, environmental factors or post-translational modifications which mimic monogenic mutations? 


\section{Glossary}

$\alpha$-synuclein ( $\alpha$-syn): The major component of Lewy bodies- the pathological hallmark of PD, and a protein with diverse roles in cellular biology and synaptic transmission. Mutations or increases in the levels of $\alpha$-syn are found in PD and increase the propensity for the protein to aggregate, a major factor in the deleterious effects ascribed to the protein.

Autophagy: A process of degradation of cellular components via breakdown in the lysosome, either by direct import: chaperone mediated autophagy (CMA) or by incorporation into autophagosomes and subsequent lysosomal fusion: micro/macroautophagy.

GWAS: Genome-wide association studies are used to identify single nucleotide polymorphisms (SNPs), which are commonly present in the population, that are associated with a phenotype, for example an increased risk of developing Parkinson's disease. GWAS can be used as a way of identifying novel mediators of disease.

Lewy bodies: intracellular protein aggregates comprised of damaged and misfolded proteins of which $\alpha$-synuclein is a prominent component. Lewy bodies are the defining pathological feature of post-mortem PD brain.

LRRK2: Mutations in Leucine-rich repeat kinase 2 (LRRK2) is the most common form of autosomal dominant Parkinson's disease.

Mitophagy: A specific form of macroautophagy, relating to degradation of mitochondria via autophagosome formation.

MPTP/MPP+: A complex I inhibitor similar in structure to dopamine. MPP+ is preferentially taken up by the dopamine transporter resulting in selective inhibition of complex I in dopaminergic neurons.

NRF2: Nuclear factor (erythroid-derived 2)-like 2 is a key transcription factor activated in response to stress, resulting in upregulation of phase II (detoxifying) enzymes including many antioxidants. 


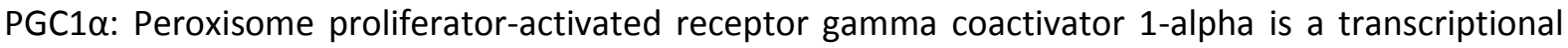
coactivator with important roles in mitochondrial biogenesis.

ROS/RNS: Reactive oxygen/nitrogen species, an umbrella term for a range of oxygen or nitrogen containing species which may damage macromolecules such as proteins, DNA or lipids.

Substantia nigra pars compacta ( $\mathrm{SNpc}$ ): The pigmented region of the midbrain in humans. The A9 region is the major site of dopaminergic cell loss in Parkinson's disease.

TH: Tyrosine hydroxylase is the rate-limiting enzyme in the synthesis of the neurotransmitter dopamine.

TOM: The translocase of the outer-mitochondrial membrane is a complex of proteins (including TOM20 and TOM40) which act to move proteins across the mitochondrial outer-membrane into the intermembrane space.

Ubiquitin: An $8.5 \mathrm{kDa}$ protein that can post-translationally modify proteins influencing their function, such as targeting proteins for degradation.

UCPs: Proteins responsible for uncoupling oxidative phosphorylation from ATP generation in mitochondria through reducing MMP. 